\title{
Demodicose na espécie Mesocricetus auratus: relato de caso
}

\author{
Diana Sousa Alcântara ${ }^{*}$, Monique Graziele Oliveira Santos ${ }^{2}$, Vicente de Paula \\ Fernandes Neto ${ }^{3}$ \\ ${ }^{1 *}$ Médica Veterinária, pós-graduanda em dermatologia veterinária pela Equalis, Universidade Federal do Piauí, Teresina, \\ Piauí, Brasil \\ ${ }^{2}$ Médica Veterinária Autônoma, pós-graduanda em dermatologia veterinária pela Equalis, Universidade Federal da Bahia, \\ Salvador, Bahia, Brasil. \\ ${ }^{3}$ Vicente de Paula Fernandes, Mestre em Ciência Animal, Universidade Federal do Piauí, Teresina, Piauí, Brasil \\ *Autor para correspondência: E-mail: diannalbr@hotmail.com
}

\begin{abstract}
RESUMO. Atualmente, a escolha de espécies exóticas como animais de estimação tem se tornado comum nos lares por serem dóceis, carinhosos, demandar poucos cuidados e ter baixo custo com a manutenção. Porém, problemas como alopecia, presença de prurido e lesões cutâneas são frequentes e geralmente inespecíficas, necessitando investigação atenta. Estes sinais podem estar relacionados a problemas diretos da pele, ou ser reflexo de alterações sistêmicas ou desequilíbrio nutricional. $\mathrm{O}$ artigo descreve um caso de um hamster da espécie Mecricetus auratus, atendido em uma clínica particular da cidade de Teresina, Piauí, apresentando crostas e prurido intenso na região dorso-lombar e prurido moderado no ouvido e andar em círculos. No raspado cutâneo e swab estéril do ouvido foram observados formas adultas de Demodex aurati e Malassezia sp. O objetivo desse trabalho é oferecer conhecimento a respeito da doença e de animais exóticos são tidos como animais de estimação. Fica evidente a importância de se conhecer a fisiologia e comportamentos de roedores domésticos para melhor avaliar as alterações patológicas.
\end{abstract}

Palavras chave: demodex, pele, roedor, sarna.

\section{Demodicosis in Mesocricetus auratus species: a case report}

\begin{abstract}
Currently, the choice of exotic species as pets has become commonplace in homes for being docile, affectionate, require little maintenance and have low cost maintenance. However, problems such as alopecia, presence of itching and skin lesions are frequent and often unspecific, requiring careful research. These signs may be related to direct skin problems, or be a reflection of systemic changes or nutritional imbalance. The article describes a case of a hamster species Mecricetus auratus, attended at a private clinic in the city of Teresina, Piauí, with crusts and intense itching in the dorso-lumbar region and moderate itching in the ear and around in circles. In skin scraping and sterile ear swab adult forms of Demodex aurati e Malassezia sp. were observed. The aim of this study is to provide knowledge about the disease and exotic animals are considered pets. It is evident the importance of understanding the physiology and behavior of domestic rodents to better evaluate the pathological changes.
\end{abstract}

Keywords: demodex, rodent, scabies, skin.

\section{Introdução}

$\mathrm{O}$ número de roedores utilizados como animais de estimação têm aumentado nos lares, entre esses animais, os hamsters são uma escolha frequente, pois são pequenos, calmos, demandam poucos cuidados e possuem baixo custo com manutenção (Quinton, 2005).

Os roedores silvestres quando criados em cativeiro podem ser acometidos por ectoparasitos que afetam a sua sanidade. Manifestações cutâneas como alopecia, eritema, crostas e prurido são comuns na clínica de pequenos animais, sendo os hamsters também afetados. A rarefação de pelos que evolui para alopecia é uma 
síndrome frequentemente observada em hamsters idosos. A alopecia também pode ser causada pelo calor excessivo e por alergia aos componentes da cama (maravalha) (Patel \& Forsythe, 2011).

O Demodex criceti e o Demodex aurati são duas espécies que se instalam na queratina e em nódulos da superfície epidérmica e nos folículos pilosos desse animal. Demodex aurati, considerado patogênico e encontrado na unidade pilo-sebácea. Estes ácaros estão associados a fatores como nutrição deficitária, imunossupressão decorrente de envelhecimento, estresse ou doença intercorrente. Os animais acometidos apresentam alopecia moderada a severa, prurido, hiperqueratinização e dermatite principalmente no dorso, membros e abdome. Demodex criceti considerado não patogênico, está presente na epiderme e é menor que o Demodex aurati, considerado patogênico e encontrado na unidade pilo-sebácea (Pessoa, 2007).

O presente trabalho tem por objetivo oferecer conhecimento sobre a doença em questão, já que a população de animais exóticos de companhia cresce cada vez mais, juntamente com a demanda por médicos veterinários especializados em cuidar deles.

\section{Material e Métodos}

Um hamster da espécie Mesocricetus auratus, foi atendido em uma clínica particular da cidade de Teresina, Piauí, apresentando crostas melicéricas e prurido intenso na região dorsolombar do abdômen (Figura 1), prurido moderado no ouvido direito e andar circular para o lado direito, que segundo a proprietária, iniciou-se após mudança de ambiente. Foram realizados raspado cutâneo e coleta de material com swab estéril no ouvido direito e esquerdo.

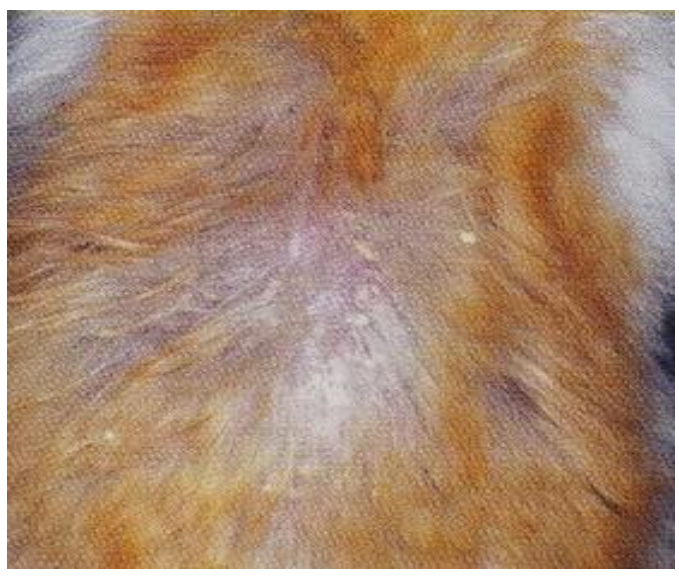

Figura 1: Crostas na região dorso-lombar

\section{Resultados e Discussão}

Foi observado no raspado cutâneo e na citologia do ouvido direito, presença de formas adultas de Demodex aurati (Figura 2A), e grande quantidade de esporos fúngicos de Malassezia sp. (Figura 2B), no ouvido direito. Para o tratamento de sarna demodécica, foi prescrita formulação comercial para gatos a base de moxidectina $0,1 \%$ e imidacloprida $1 \%$, uma gota na região da nuca, 1x por semana, por quatro semanas e formulação comercial a base de diazinon $4 \%$ para $\mathrm{o}$ tratamento de sarna otodécicano ouvido.

Segundo Moraillon et al. (2013), demodex são parasitas de roedores e lagomorfos, e eles são específicos para cada espécie. O Demodex aurati é o ácaro de causa mais comum e mais patogênico em hamsters, e se caracteriza por ser um parasito dos folículos pilosos, de formato alongado, patas atrofiadas, que geralmente causa lesões alopécicas difusas com seborréia, descamação e hiper pigmentação, características que foram observadas no presente relato. $\mathrm{O}$ parasitismo por demodex em roedores não ocorre apenas nas áreas corporais estudadas no presente trabalho, mas em regiões distintas descritas por outros autores, dentre elas: área conjuntiva (Ronald \& Wagner, 1976), superfícies da genitália, focinho, dorso da cabeça (Nutting, 1961), glândulas prepucial, clitorial (Bukva, 1984) e membros posteriores (Schönfelder et al., 2010). Resultados positivos para Demodex aurati na região dorso-lombar de roedores foi igualmente verificado por no Japão.

Os animais pertencentes á espécie Mesocricetus auratus, são os menos resistentes e os mais frágeis de todos os pequenos roedores domésticos, sendo muito suscetíveis a doenças causadas pelo stress, como a ileíte proliferativa e a sarna demodécica. Os hamsters são também muito sensíveis ao calor e à umidade e devem ser mantidos em locais frescos (Quesenberry \& Carpenter, 2011), sendo assim, levando em consideração essas características, pode-se sugerir que o aparecimento da sarna demodécica foi decorrente de uma imunossupressão devido ao estresse ocasionado pela mudança de ambiente sofrida pelo animal, já que o mesmo não possui nenhuma doença base e nem alimentação deficiente.

No que diz respeito ao tratamento da demodicose, Moraillon et al. (2013) citam que o mais eficaz passa pela administração de banhos de amitraz ou pela a aplicação de uma solução 
tópica de $0,05 \%$ de amitraz, duas vezes por semana, até não se observarem ácaros nas colheitas por raspagem de pele. Todavia, o amitraz pode apresentar toxicidade em porquinhos-da-índia e hamsters, devendo ser utilizada com precaução. A moxidectina (Adovocate ${ }^{\circledR}$ ) pode ser utilizada em alternativa aos outros tratamentos, devendo ser aplicada em solução "spot-on", a uma dose de $1 \mathrm{mg} / \mathrm{kg}$, uma vez por semana, sendo a molécula mais interessante atualmente, o que influenciou diretamente na escolha da mesma para o tratamento, demonstrando ser a opção que mais leva em conta a segurança do animal e a praticidade da utilização da medicação pela tutora, podendo a mesma realizá-la em casa, evitando estresse ao animal devido ao deslocamento e manipulação da medicação no consultório e menor risco de intoxicação ou efeitos colaterais.

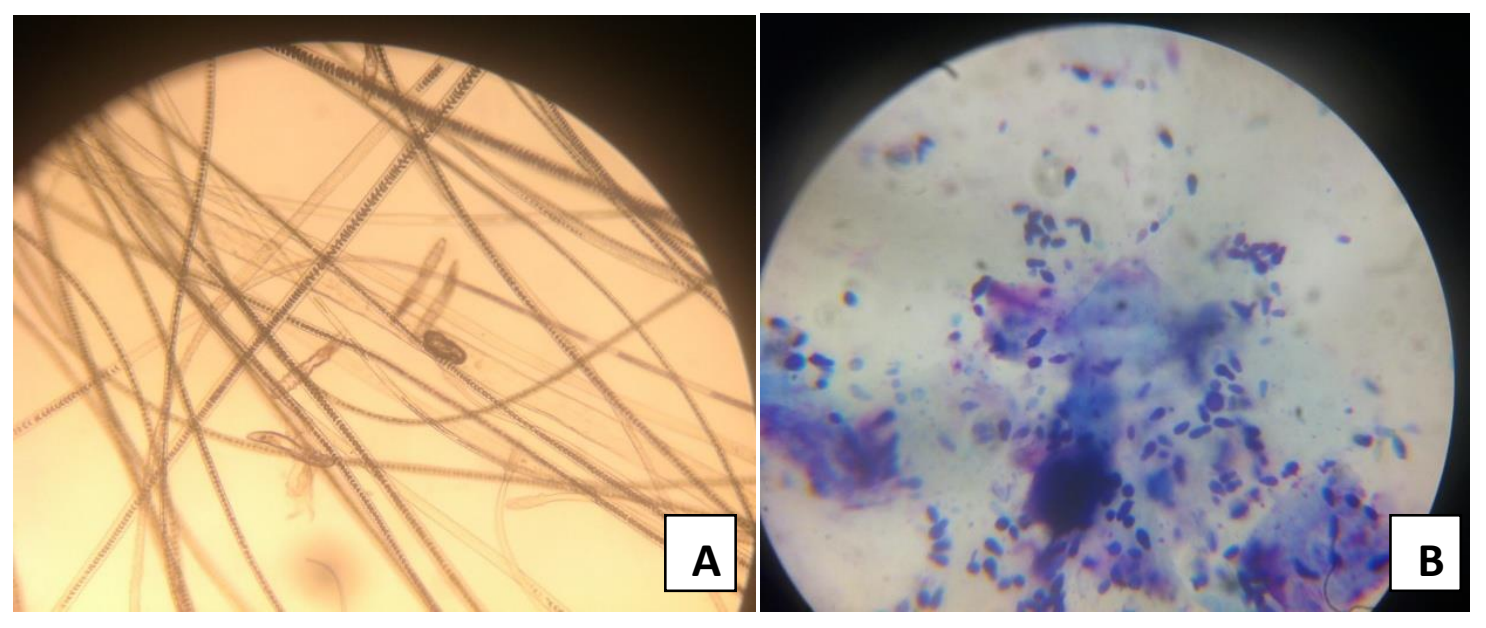

Figura 2. A-Demodex aurati; B - Malassezia sp. no ouvido direito

A presença de Demodex aurati nos swabs do pavilhão auricular é justificada uma vez que o cerúmen nos roedores, cera de cor amareloamarronzada e de consistência oleosa, é considerado um sítio auricular de sobrevivência para esse parasito (Pessoa, 2007). Além disso, o conduto auditivo apresenta uma variedade de micro hábitat para o demodex, dentre eles pode ser mencionado às glândulas sebáceas, nas quais esse ácaro habita suas paredes secretoras e excretoras (Guimarães et al., 2001). Em hamster, o ácaro específico da espécie e associado a infecções no meato acústico, é o Notoedres notoedres (Quinton, 2005). A otite é uma enfermidade multifatorial resultante do processo inflamatório da cavidade auditiva tendo origem na região externa e podendo prolongar-se até o sistema vestíbulo-coclear. Esse processo migratório geralmente é acompanhado por complicações mais graves e extensas (Greene et al., 1993). Outro agente causador de problemas otológicos é a Malassezia pachydermatis, uma levedura que faz parte da micro biota do conduto auditivo, de natureza oportunista, tornando-se patogênica quando há uma alteração no ambiente microbiológico da superfície da pele ou no sistema imunológico do hospedeiro levando a um quadro de otite (Meredith, 2006).

\section{Conclusão}

Diante do caso apresentado, fica evidente a necessidade de se conhecer cada vez mais os aspectos fisiológicos e comportamentais de roedores domésticos, para melhor orientar e avaliar quando há alterações patológicas, já que atualmente a procura pelos mesmos como animais de estimação tem aumentado, e um diagnóstico rápido e preciso é importante para estabelecer o tratamento correto, além do cuidado com a medicação e doses utilizadas.

\section{Referências Bibliográficas}

Bukva, V. (1984). Demodex flagellurus sp. n. (Acari: Demodicidae) from the preputial and clitoral glands of the house mouse, Mus musculus L. Folia Parasitologica, 32, 73-81.

Greene, C. E., Samperio, J. O. \& Gómez, J. P. (1993). Enfermedades infecciosas: Perros y gatos. Editora Interamericana, São Paulo. 
Guimarães, J. H., Battesti, D. M. B. \& Tucci, E. C. (2001). Ectoparasitos de importância veterinária. FAPESP, São Paulo.

Meredith, A. (2006). Skin diseases and treatment of guinea pigs. Skin Diseases of Exotic Pets, 232-251.

Moraillon, R., Legeay, Y., Boussarie, D. \& Sénécat, O. (2013). Manual Elsevier de Veterinária: Diagnóstico e tratamento de Cães, gatos e animais exóticos, 7th edn. Elsevier, Rio de Janeiro.

Nutting, W. B. (1961). Demodex aurati sp. nov. and D. criceti, ectoparasites of the golden hamster (Mesocricetus auratus). Parasitology, 51, 515-522.

Patel, A. \& Forsythe, P. J. (2011). Dermatologia em pequenos animais. Elsevier Brasil, Rio de Janeiro.

Pessoa, C. A. (2007). Rodentia-Roedores de Companhia (Hamster, Gerbil, Cobaia, Chinchila e Rato). Roca, São Paulo.

Quesenberry, K. \& Carpenter, J. W. (2011). Ferrets, rabbits and rodents: clinical medicine and surgery. Elsevier Health Sciences, St. Louis.

Quinton, J. F. (2005). Novos Animais de Estimação-Pequenos Mamíferos. Editora Roca, São Paulo.

Ronald, N. C. \& Wagner, J. E. (1976). Arthropod parasites of the genus Cavia. JE Wagner \& PJ Manning, eds, New York.

Schönfelder, J., Henneveld, K., Schönfelder, A., Hein, J. \& Müller, R. (2010). Concurrent infestation of Demodex caviae and Chirodiscoides caviae in a guinea pig. Tierärztliche Praxis Kleintiere, 38, 28-30.

\section{Article History:}

Received 15 April, 2016

Accepted 16 May, 2016

Available online 1 June, 2016

License information: This is an open-access article distributed under the terms of the Creative Commons Attribution License, which permits unrestricted use, distribution, and reproduction in any medium, provided the original work is properly cited 